\title{
Stress Wave Propagation in the Ground Containing a Spherical Cavity*
}

\author{
Shinji TANIMURA**, Kouichi KAIZU*** and Fusao OKA****
}

\begin{abstract}
The governing equations of dynamic problems of elastic-viscoplastic three-dimensional soil containing spherical cavities are formulated by means of bipolar coordinates, adapting constitutive equations of normally consolidated clay. The stress wave propagation in the ground containing a spherical cavity of $0.5 \mathrm{~m}$ diameter at a $5 \mathrm{~m}$ depth is analyzed by the method of direct integration of the basic equations of the problem, when an axisymmetric dynamic load is applied to the boundary of the cavity. In the calculation, the change in pressure with depth from the ground surface is also taken into account for the initial state of the soil.
\end{abstract}

Key Words: Numerical Analysis, Soil Mechanics, Stress Wave, Elastic-Viscoplastic Soil, Bipolar Coordinates

\section{Introduction}

From the viewpoint of improving the security of a structure in the ground and from the fact that some studies have been done for the purpose of storing a large quantity of fuel under the ground, the importance of studying the dynamic behavior of the ground may increase in the future. Elastic wave propagation in the ground has been studied since olden times. It seems, however, that the three-dimensional dynamic problem of the ground containing spherical cavities, by modeling the soft ground as an elastic-viscoplastic medium, has not been studied.

In this paper, the governing equations of dynamic problems of three-dimensional soft ground containing

* Received 17th September, 1986. Paper No. 85-0620A

** Department of Mechanical Engineering, University of Osaka Prefecture, 4-804 Mozu, Sakai, Osaka, 591, Japan

**** Osaka Prefectural Technical College, Saiwaimachi, Neyagawa, Osaka, 572, Japan

**** Department of Civil Engineering, Gifu University, Yanagido, Gifu, 501-11, Japan spherical cavities are formulated by means of bipolar coordinates $^{(1)(2)}$. Practical constitutive equations for an elastic-viscoplastic medium, which simulate the soft ground of normally consolidated clays, are used. As numerical examples, stress wave propagation in the ground containing a spherical cavity of $0.5 \mathrm{~m}$ diameter at a $5 \mathrm{~m}$ depth is analyzed by the method of direct integration of the basic equations of the problems, when an axisymmetric dynamic load is applied to the boundary of the cavity. In the calculation, the change in pressure with depth from the ground surface is also taken into account for the initial state of the soil.

\section{Governing Equations}

Let us assume a semi-infinite ground with a spherical cavity at a distance $h$ from the ground surface (the plane boundary), and a dynamic load applied to the boundary (the ground surface or the inside of the cavity). In this case, it is not required that the load distribution be axisymmetric.

Now, let us introduce bipolar coordinates $(\alpha, \beta$, $\varphi)^{(1)(2)}$, where points $\mathrm{O}_{1}$ and $\mathrm{O}_{2}$ are at $X= \pm a=\sqrt{h_{r}^{2}-1}$ 


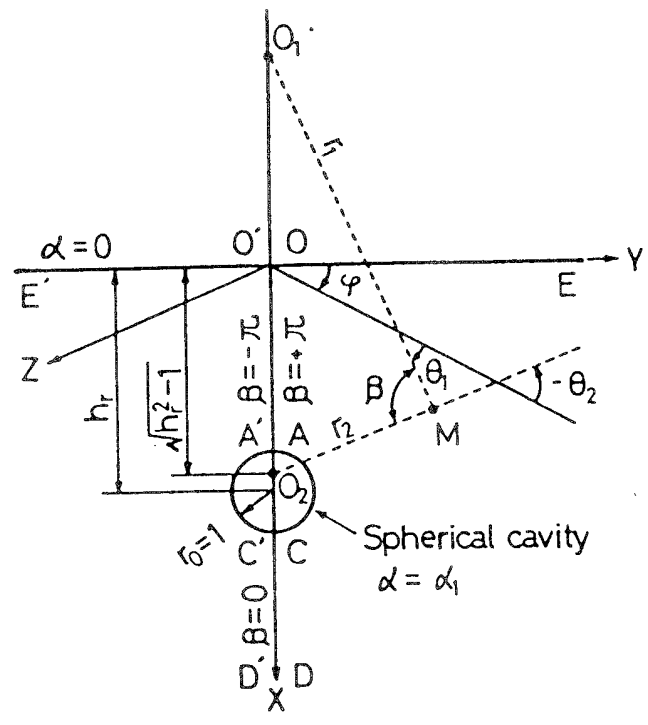

Fig. 1 Coordinate system

as shown in Fig. 1, and constitute the poles for the transformation from rectangular coordinates $(X, Y$, $Z$ ), where $h_{r}=h / r_{0}$ is the distance from the origin to the center of the cavity and $r_{0}$ is the radius of the spherical cavity (the unit lenght). We use practical constitutive equations ${ }^{(3)}$ for elastic-viscoplastic bodies, which simulate the soft ground of normally consolidated clays.

In curvilinear orthogonal coordinate systems, the constitutive equations are expressed in contravariant components as follows:

$$
\begin{aligned}
d^{i j} & =\frac{1}{2 G} \dot{s}^{i j}+\frac{1}{9 K} \dot{\sigma}_{k}{ }^{k} g^{i j}+C\langle\Phi(F)\rangle \frac{s^{i j}}{\sqrt{2 J_{2}}} \\
& +\frac{C}{3}\langle\Phi(F)\rangle\left(M^{*}-\frac{\sqrt{2 J_{2}}}{\sigma_{m}^{\prime}}\right) g^{i j} \quad\left(\sigma_{k}{ }^{k} \leqq 0\right),
\end{aligned}
$$

where it is assumed that the deformation is infinitesimal and the spin tensor is not of greater order than the deformation rate tensor, and therefore the deformation-rate tensor of Euler $d_{i j}$ is used instead of the strain rate tensor $\varepsilon_{i j}$. The symbol $\langle\phi(F)\rangle$ is defined as

$$
\begin{aligned}
& \langle\Phi(F)\rangle=0 \text { for }(F \leqq 0) \\
& \langle\Phi(F)\rangle=\Phi(F) \text { for }(F>0),
\end{aligned}
$$

and the symbol $\Phi(F)$ is expressed as follows:

$$
\Phi(F)=\exp \left\{m^{\prime}\left(\frac{\sqrt{2 J_{2}}}{M^{*} \sigma_{m}^{\prime}}+\ln \frac{\sigma_{m}^{\prime}}{\sigma_{m e}^{\prime}}+\frac{1+e}{\lambda-x} v^{p}\right)\right\},
$$

where

$$
\begin{aligned}
& \sigma_{m}^{\prime}=\sigma_{k}{ }^{k} / 3=\sigma_{k}{ }^{k} / 3-u, \sigma^{i j^{\prime}}=\sigma^{i j}-u g^{i j}, \\
& s^{i j}=\sigma^{i j}-\sigma_{k}{ }^{i j} g^{i j} / 3, \sigma_{k}{ }^{k}=g_{k m} \sigma^{k m} \\
& J_{2}=s^{i j} s_{i j} / 2=s^{i j} s^{k l} g_{k i} g_{t j} / 2 \\
& K=-(1+e) \sigma_{m}^{\prime} /(1-B) x \\
& G=\frac{1683(2.97-e)^{2}}{1+e} \sqrt{-\sigma_{m}^{\prime}}
\end{aligned}
$$

(for Kyoto Fukakusa clay when the values of $G$ and $\sigma_{m}^{\prime}$ are expressed in $\mathrm{kPa}$ and $\left.\sigma_{m}^{\prime} \leqq-98.07\right)$, and the quantities $\sigma_{i j}, \varepsilon_{i j}, s_{i j}, J_{2}, g_{i j}$ and $g^{i j}$ are the stress tensor, strain tensor, deviatoric stress tensor, second invariant of the stress deviator, covariant metric tensor and contravariant metric tensor, respectively, and $\sigma_{m}^{\prime}$ is the mean effective stress, $\sigma_{m e}^{\prime}$ denotes one of the effective stress states reached after an arbitary time duration of consolidation, $u$ is the pore water pressure, $v^{p}$ is the plastic volumetric strain $\left(=\varepsilon_{k}^{p k}\right), e$ is a void ratio, $\mathrm{B}$ is the pore pressure coefficient, and the quantities $\mathcal{x}, \lambda, M^{*}, C$ and $m^{\prime}$ are the material parameters.

Since bipolar coordinates $(\alpha, \beta, \varphi)$ are ones of the curvilinear orthogonal coordinate system, we can obtain the governing equations using the equations of motion and the constitutive equations expressed in the curvilinear orthogonal coordinate system, in the same manner presented in the previous paper ${ }^{(1)(2)}$. The dimensional factor may be eliminated by writing

$$
\begin{aligned}
& U=\frac{v^{\alpha}}{a_{1}}, V=\frac{v^{\beta}}{a_{1}}, R=\frac{v^{\varphi}}{a_{1}}, \\
& P=\frac{\sigma^{\alpha \alpha}}{\rho a_{1}^{2}}, Q=\frac{\sigma^{\beta \beta}}{\rho a_{1}^{2}}, S=\frac{\sigma^{\varphi \varphi}}{\rho a_{1}^{2}}, \\
& T=\frac{\sigma^{\alpha \beta}}{\rho a_{1}^{2}}, M=\frac{\sigma^{\alpha \varphi}}{\rho a_{1}^{2}}, N=\frac{\sigma^{\beta \varphi}}{\rho a_{1}^{2}}, \\
& P_{i}=\frac{\left(\sigma^{\alpha \alpha}\right)_{i}}{\rho a_{1}^{2}}, Q_{i}=\frac{\left(\sigma^{\beta \beta}\right)_{i}}{\rho a_{1}^{2}}, S_{i}=\frac{\left(\sigma^{\varphi \varphi}\right)_{i}}{\rho a_{1}^{2}}, \\
& P_{u}=\frac{\left(\sigma^{\alpha \alpha}\right)_{u i}}{\rho a_{1}^{2}}, Q_{u}=\frac{\left(\sigma^{\beta \beta}\right)_{u i}}{\rho a_{1}^{2}}, \\
& S_{u}=\frac{\left(\sigma^{\varphi \varphi}\right)_{u i}}{\rho a_{1}^{2}}, \Gamma=\frac{a_{1}}{a_{2}}, \tau=\frac{a_{1}}{\gamma_{0}} t,
\end{aligned}
$$

where $\sigma^{i j}$ are the contravariant components of the stress tensor, $v^{i}$ are the contravariant components of the velocity vector, $\rho$ is the density of the medium, and $a_{1}=\{(3 K+4 G) / 3 \rho\}^{1 / 2}, a_{2}=(G / \rho)^{1 / 2}$,

where $a_{1}$ and $a_{2}$ are the velocities of dilatation and distortion waves. The index " $i$ " denotes the initial state of the stress for earth pressure, $u_{i}$ denotes the initial state for pore pressure, and $\left(\sigma^{\alpha \alpha}\right)_{u i},\left(\sigma^{\beta \beta}\right)_{u i}$ and $\left(\sigma^{\varphi \varphi}\right)_{u i}$ are the components of the stress tensor expressing the initial state of the stress for pore pressure. Moreover let us express as follows:

$$
\sqrt{2 \widetilde{J}_{2}}=\sqrt{2 J_{2}} / \rho a_{1}^{2},\langle\widetilde{\Psi}\rangle=\langle\Phi(F)\rangle, \Lambda=C / a_{1} .
$$

Omitting the details of the deriving process, we can obtain the systems of the governing equations as follows:

$$
L[U]=A U_{, \tau}+B U_{, \alpha}+C U_{, \beta}+D U_{, \varphi}+E=0,
$$

where vectors $U$ and $E$ are given by

$$
\begin{aligned}
& U^{r}=(U, V, R, T, M, N, P, Q, S), \\
& E^{T}=\left(\psi_{1}, \psi_{2}, \psi_{3}, \psi_{4}, \psi_{5}, \psi_{6}, \psi_{7}, \psi_{8}, \psi_{9}\right),
\end{aligned}
$$

$A, B, C$ and $D$ are nine-dimensional square matrices which are the same as those presented in the previous paper $^{(1)(2)}$. The components of vector $\boldsymbol{E}$ are as follows: 


$$
\begin{aligned}
& \psi_{1}=-\left[\frac{1}{\cosh \alpha-\cos \beta}\left\{\sinh \alpha\left(U^{2}-V^{2}-\sin ^{2} \beta R^{2}-4 P+Q+\sin ^{2} \beta S\right)+2 \sin \beta U V\right\}\right. \\
& \left.+\frac{1}{\sin \beta}\left(\cos \beta-\frac{5 \sin ^{2} \beta}{\cosh \alpha-\cos \beta}\right) T\right] \\
& \psi_{2}=\frac{1}{\cosh \alpha-\cos \beta}\left\{\sin \beta\left(U^{2}-V^{2}-P-Q\right)-\sinh \alpha(2 U V-5 T)\right\} \\
& +\sin \beta\left(\cos \beta-\frac{\sin ^{2} \beta}{\cosh \alpha-\cos \beta}\right)\left(S-R^{2}\right)-\frac{1}{\sin \beta}\left(\cos \beta-\frac{5 \sin ^{2} \beta}{\cosh \alpha-\cos \beta}\right) Q, \\
& \psi_{3}=\frac{\sinh \alpha}{\cosh \alpha-\cos \beta}(5 M-2 U R)+\frac{2}{\sin \beta}\left(\cos \beta-\frac{\sin ^{2} \beta}{\cosh \alpha-\cos \beta}\right) V R \\
& -\frac{1}{\sin \beta}\left(3 \cos \beta-\frac{5 \sin ^{2} \beta}{\cosh \alpha-\cos \beta}\right) N \text {, } \\
& \psi_{4}=2 g^{11} \Lambda\langle\tilde{\Psi}\rangle T / \sqrt{2 \tilde{\tilde{J}_{2}}}, \\
& \psi_{5}=2 g^{11} \Lambda\langle\widetilde{\Psi}\rangle M / \sqrt{2 \widetilde{J}_{2}}, \\
& \psi_{6}=2 g^{11} \Lambda\langle\tilde{\Psi}\rangle N / \sqrt{2 \tilde{J}_{2}} \text {, } \\
& \psi_{7}=-\left[\frac{3 \Gamma^{2}-4}{\Gamma^{2}} \frac{\sinh \alpha}{\cosh \alpha-\cos \beta} g^{11} U+\left\{\frac{2\left(\Gamma^{2}-1\right)}{\Gamma^{2}} \frac{\sin \beta}{\cosh \alpha-\cos \beta}\right.\right. \\
& \left.+\frac{2-\Gamma^{2}}{\Gamma^{2}} \frac{1}{\sin \beta}\left(\cos \beta-\frac{\sin ^{2} \beta}{\cosh \alpha-\cos \beta}\right)\right\} g^{11} V+\frac{3 \Gamma^{2}-4}{3 \Gamma^{2}} \Lambda\langle\tilde{\Psi}\rangle\left\{M^{*}-\frac{3 \sqrt{2 \tilde{J}_{2}}}{(1-B) \sum+B \Sigma_{i}-\Sigma_{u}}\right\} g^{11} \\
& \left.+\frac{2}{3 \Gamma^{2}} \Lambda\langle\tilde{\Psi}\rangle\left(2 P-Q-g_{33} g^{11} S\right) \frac{1}{\sqrt{2 \widetilde{J}_{2}}}\right] \text {, } \\
& \psi_{8}=-\left[\frac{3 \Gamma^{2}-4}{\Gamma^{2}} \frac{\sinh \alpha}{\cosh \alpha-\cos \beta} g^{11} U+\left\{\frac{2\left(\Gamma^{2}-1\right)}{\Gamma^{2}} \frac{\sin \beta}{\cosh \alpha-\cos \beta}\right.\right. \\
& \left.+\frac{2-\Gamma^{2}}{\Gamma^{2}} \frac{1}{\sin \beta}\left(\cos \beta-\frac{\sin ^{2} \beta}{\cosh \alpha-\cos \beta}\right)\right\} g^{11} V+\frac{3 \Gamma^{2}-4}{3 \Gamma^{2}} \Lambda\langle\tilde{\Psi}\rangle\left\{M^{*}-\frac{3 \sqrt{2 \widetilde{J}_{2}}}{(1-B) \Sigma+B \Sigma_{i}-\Sigma_{u}}\right\} g^{11} \\
& \left.+\frac{2}{3 \Gamma^{2}} \Lambda\langle\widetilde{\Psi}\rangle\left(-P+2 Q-g_{33} g^{11} S\right) \frac{1}{\sqrt{2 \widetilde{J}_{2}}}\right], \\
& \psi_{9}=-\left[\frac{3 \Gamma^{2}-4}{\Gamma^{2}} \frac{\sinh \alpha}{\cosh \alpha-\cos \beta} g^{33} U+\left\{\frac{2\left(\Gamma^{2}-2\right)}{\Gamma^{2}} \frac{\sin \beta}{\cosh \alpha-\cos \beta}\right.\right. \\
& \left.-\frac{1}{\sin \beta}\left(\cos \beta-\frac{\sin ^{2} \beta}{\cosh \alpha-\cos \beta}\right)\right\} g^{33} V+\frac{3 \Gamma^{2}-4}{3 \Gamma^{2}} \Lambda\langle\tilde{\Psi}\rangle\left\{M^{*}-\frac{2 \sqrt{3 \tilde{J}_{2}}}{(1-B) \Sigma+B \sum_{i}-\Sigma_{u}}\right\} g^{33} \\
& \left.+\frac{2}{3 \Gamma^{2}} \Lambda\langle\tilde{\Psi}\rangle\left(-g_{11} g^{33} P-g_{11} g^{33} Q+2 S\right) \frac{1}{\sqrt{2 \widetilde{J}_{2}}}\right] \text {, }
\end{aligned}
$$

where

$$
\begin{aligned}
& \langle\widetilde{\Psi}\rangle=\left\langle\operatorname { e x p } \left\{m ^ { \prime } \left[\frac{3 \sqrt{2 \tilde{J}_{2}}}{M^{*}\left\{(1-B) \sum+B \sum_{i}-\sum_{u}\right\}}\right.\right.\right. \\
& \left.\left.\left.\quad+\ln \frac{\left\{(1-B) \sum+B \sum_{i}-\sum_{u}\right\}}{\sum_{i}-\sum_{u}}+\frac{1+e}{\lambda-\chi} v^{p}\right]\right\}\right\rangle \\
& 2 \tilde{J}_{2}=\left(P^{2}+2 T^{2}+Q^{2}\right) g_{11}^{2}+2\left(M^{2}+N^{2}\right) g_{11} g_{33} \\
& \quad+S^{2} g_{33}^{2}-\left(g_{11} P+g_{11} Q+g_{33} S\right)^{2} / 3 \\
& \sum^{2}=g_{11} P+g_{11} Q+g_{33} S \\
& \sum_{i}=g_{11} P_{i}+g_{11} Q_{i}+g_{33} S_{i} \\
& \sum_{u}=g_{11} P_{u}+g_{11} Q_{u}+g_{33} S_{u} .
\end{aligned}
$$

\section{Model of the Soft Ground}

As shown in Fig. 2, we deal with stress wave propagation in the ground containing a spherical cavity of radius $r_{0}$ at a distance $h$ from the ground surface. In this section, we model the soft ground in an initial state and simulate the dynamic properties of the ground using rectangular coordinates $(x, y)$.

\section{1 Initial conditions}

Let us suppose the ground consists of fully saturated, normally consolidated clay which consists

of solid and liquid phases. The change in pressure with depth from the ground surface can be also taken into account. The initial pressure $\sigma_{k k}^{(s)}(x) / 3[\mathrm{kPa}]$ of point

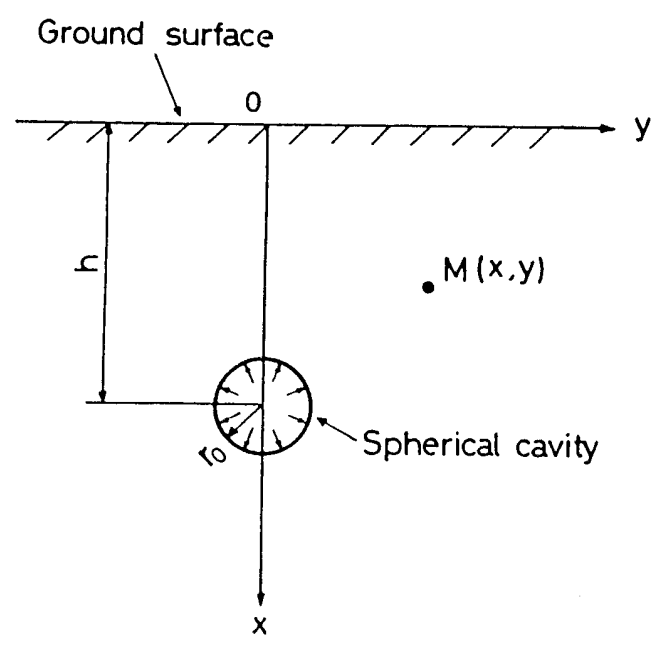

Fig. 2 Ground containing a spherical cavity 
$\mathrm{M}(x, y)$ (Fig.2) at a distance $x[\mathrm{~m}]$ from the ground surface is given by

$$
\frac{1}{3} \sigma_{k h}^{(s)}(x)=-\left(98.07+9.807 \frac{G_{s}+e}{1+e} x\right)
$$

where $9.807\left(G_{s}+e\right) /(1+e)$ is the specific weight of the clay containing water, and $G_{s}$ is the specific gravity of the soil particles. The first term on the right side of the equation, "-98.07", which is the component of atmospheric pressure, is added for convenience's sake, because the constitutive equations for consolidated clays are used, so the numerical computation becomes difficult if $\sigma_{m}^{\prime}=0$ is assumed on the ground surface ( $x$ $=0)$. The index " $(s)$ " denotes the static state.

On the supposition that $u^{(s)}(x)[\mathrm{kPa}]$, which denotes the initial state of the pore water pressure, is given by the hydraulic pressure at depth of water $x$ [m], we have as follows:

$$
u^{(s)}(x)=-9.807 x \text {. }
$$

In the initial condition, the mean effective stress of point $\mathrm{M}, \sigma_{m}^{\prime(s)}(x)\left[=\sigma_{m e}^{\prime}(x)\right][\mathrm{kPa}]$, is expressed using the relation $\sigma_{m}^{\prime(s)}(x)=\sigma_{k k}^{(s)}(x) / 3-u^{(s)}(x)$, as follows:

$$
\sigma_{m}^{\prime(s)}(x)=\sigma_{m e}^{\prime}(x)=-\left(98.07+9.807 \frac{G_{s}-1}{1+e} x\right),
$$

The plastic volumetric strain is given by

$$
v^{p}=-\frac{\lambda-x}{1+e} \ln \left(\frac{\sigma_{m y}^{\prime(s)}}{\sigma_{m y i}^{\prime(s)}}\right),
$$

where $\sigma_{m y}^{\prime(s)}$ is the static hardening parameter and $\sigma_{m y i}^{\prime s)}$ is the initial, static hardening parameter. We assume the following relations in order to express the plastic volumetric strain $v_{0}^{P}(x)$ in the initial state,

$$
\begin{aligned}
& \sigma_{m y i}^{\prime(s)} \fallingdotseq \sigma_{m}^{\prime(s)}(0)=-98.07[\mathrm{kPa}], \\
& \sigma_{m y}^{\prime(s)} \fallingdotseq \sigma_{m}^{\prime(s)}(x),
\end{aligned}
$$

and then we obtain

$$
v_{0}^{p}(x)=-\frac{\lambda-x}{1+e} \ln \left(1+\frac{G_{s}-1}{1+e} \frac{x}{10}\right) .
$$

\section{2 Under dynamic loads}

The plastic volumetric strain $v^{p}(x)$ of point $\mathrm{M}$ at an arbitary time $t$ is given by

$$
v^{p}(x)=v_{0}^{p}(x)+\int_{0}^{t} \dot{\varepsilon}_{k k}^{p}(x) d t .
$$

When stress waves propagate under dynamic loading, the soil may be considered to be under the undrained condition. Under this condition, the following relation between the change in stress, which denotes the change in pore water pressure, $\Delta u$, and the change in total stress, $\Delta \sigma$, may be given by

$$
\Delta u=B \Delta \sigma \text {, }
$$

where $B$ is the pore water pressure coefficient, and $B$ $\fallingdotseq 1$ when the soil is fully saturated and undrained. Using this relation, we can write as follows:

$$
\begin{aligned}
& u(x)-u^{(s)}(x)=B\left\{\sigma_{k k}(x) / 3-\sigma_{k h}^{(s)}(x) / 3\right\} \\
& \left(\sigma_{k h} \leqq 0\right) .
\end{aligned}
$$

Then, the mean effective stress $\sigma_{m}^{\prime}(x)[\mathrm{kPa}]$ of point $\mathrm{M}$ under dynamic loading is expressed, using the relation $\sigma_{m^{\prime}}(x)=\sigma_{k k}(x) / 3-u(x)$ as follows:

$$
\begin{aligned}
& \sigma_{m^{\prime}}(x)=(1-B) \sigma_{k k}(x) / 3 \\
& \quad+B \sigma_{k k}^{(s)}(x) / 3-u^{(s)}(x) \quad\left(\sigma_{k k} \leqq 0\right) .
\end{aligned}
$$

\section{Numerical Examples}

As shown in Fig. 2, we analyzed the stress wave propagation in the ground containing a spherical cavity of $0.5 \mathrm{~m}$ diameter at a $5 \mathrm{~m}$ depth, when an axisymmetric dynamic load is applied to the inside of the cavity. For axisymmetric problems, $\varepsilon^{\alpha \varphi}=\varepsilon^{\beta \varphi}=0$, $\sigma^{\alpha \varphi}=\sigma^{\beta \varphi}=0$ and $v^{\varphi}=0$, because the displacement vector $u^{\varphi}=0$. Therefore, it is sufficient to restrict the discussion to the $\varphi=0$ plane. The non-vanishing components of the stress tensor and velocity vector are $\sigma^{\alpha \alpha}, \sigma^{\alpha \beta}, \sigma^{\beta \beta}, \sigma^{\phi \varphi}, v^{\alpha}$ and $v^{\beta}$. The system of the governing equations for these axisymmetric problems can be obtained by putting $\mathrm{R}=\mathrm{M}=\mathrm{N}=0$ and the derivatives with respect to $\partial / \partial \varphi$ in Eqs. (5), (6), (7) and (8) equal to zero.

\section{1 Initial conditions}

The components of the stress tensor $\left(\sigma^{\alpha \alpha}\right)_{i},\left(\sigma^{\beta \beta}\right)_{i}$ and $\left(\sigma^{\phi \varphi}\right)_{i}$, which denote the initial pressure of the earth pressure at $\tau=0$, are obtained by transforming the value of $\sigma_{k k}^{(s)} / 3$ given by Eq. (9) into values in rectangular coordinates $(X, Y, Z)$, and by transforming them into values in bipolar coordinates $(\alpha, \beta, \varphi)$. The components of the stress tensor $\left(\sigma^{\alpha \alpha}\right)_{u i},\left(\sigma^{\beta \beta}\right)_{u i}$ and $\left(\sigma^{\varphi \varphi}\right)_{u i}$, which denote the initial state of the pore water pressure, are obtained by transforming the value of $u^{(s)}$ given by Eq. (10) into values in bipolar coordinates, in the same manner. Similarly, the initial value of the plastic volumetric strain is given by Eq. (14). The particle velocities are zero at $\tau=0$.

\section{2 Boundary conditions}

We deal with the case when an axisymmetric dynamic pressure with respect to the O'X axis is applied to the inside of the cavity $\left(\alpha=\alpha_{1}\right)$ and assume the following:

$$
\begin{aligned}
& P\left(\alpha_{1}, \beta, \tau\right)=-\frac{(\cosh \alpha-\cos \beta)^{2}}{\rho a_{1}^{2} a^{2}} \\
& \quad \times\left\{2\left(p_{m}-p_{i}\right) \frac{\tau_{m} \tau}{\tau^{2}+\tau_{m}^{2}}+p_{i}\right\}, \\
& T\left(\alpha_{1}, \beta, \tau\right)=0,
\end{aligned}
$$

where $p_{m}$ is the maximum pressure, $p_{i}$ is the initial pressure on the boundary of the cavity, $\tau_{m}=a_{1} t_{m} / \gamma_{0}$, and $t_{m}$ is the time when the dynamic pressure reaches the maximum pressure.

On the plane boundary $(\alpha=0)$, the following conditions hold,

$$
P(0, \beta, \tau)=-\frac{(1-\cos \beta)^{2}}{\rho a_{1}^{2} a^{2}} p_{i}, \quad T(0, \beta, \tau)=0,
$$


where $p_{i}$ is the atmospheric pressure, and on the $\mathrm{O}^{\prime} \mathrm{X}$ axis:

$$
\left.\begin{array}{l}
V(\alpha, 0, \tau)=0, T(\alpha, 0, \tau)=0, \\
V(\alpha,-\pi, \tau)=0, T(\alpha,-\pi, \tau)=0, \\
U_{, \beta}=P_{, \beta}=Q_{, \beta}=0 .
\end{array}\right\}
$$

\section{3 Numerical procedure}

We restrict the discussion to the $\varphi=0$ plane for the axisymmetric problems and to the region $\beta \leqq 0$ in the $\alpha \beta$ plane. Numerical results can be obtained in the same manner as in the previous paper ${ }^{(1)(2)}$. That is, the $\alpha \beta$ plane is divided into a rectangularly-latticed net of straight lines with $\alpha=$ const. and $\beta=$ const., and the problem is solved by the method of direct integration of the governing equations. The equations of the poblems are integrated with respect to time by the use of the Runge-Kutta-Gill method, by dividing the time axis into equal intervals. By carrying out numerical computations, changing the combination of the values of $\Delta \alpha, \Delta \beta$ and $\Delta \tau$, we can confirm the stability of the numerical solutions. In the process of computing, the following conditions are used by inspecting the value of [ ] in the first equation of Eq. (8) at each mesh point,

$$
\langle\tilde{\Psi}\rangle=0 \text { for }[\quad] \leqq 0,\langle\tilde{\Psi}\rangle=\tilde{\Psi} \text { for }[\quad]>0 .
$$

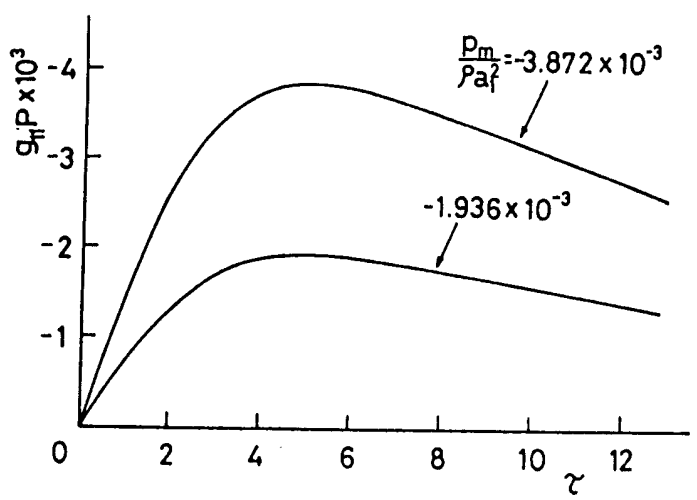

Fig. 3 Dynamic pressure on the boundary of the cavity

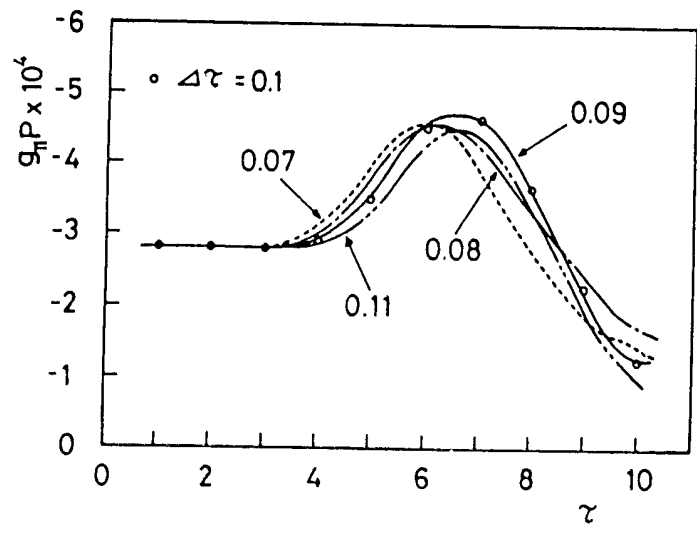

Fig. 4 Stability of the numerical solutions for $\Delta \tau$

\section{4 Parameters}

The depth from the ground surface to the center of the spherical cavity was chosen as $h_{r}=10$. The values of the parameters and the coefficients were chosen as $e=0.72, x=0.02, \lambda=0.1, M^{*}=-1.225, m^{\prime}=$ 10.0, $C=1.0 \times 10^{-16}(1 / \mathrm{s}), G_{s}=2.64$ and $B=0.9766 . G$ and $K$ are functions of $\sigma_{m}^{\prime}$, but for the sake of simplicity, constant values of a point at $h_{r} / 2(2.5 \mathrm{~m}$ depth from the ground surface) of $K=4.338 \times 10^{5} \mathrm{kPa}, G=$ $5.461 \times 10^{4} \mathrm{kPa}, a_{1}=509.3 \mathrm{~m} / \mathrm{s}, a_{2}=167.2 \mathrm{~m} / \mathrm{s}$ and $\rho a_{1}^{2}=$ $5.066 \times 10^{5} \mathrm{kPa}$ were used.

The coefficients which specify the distribution of the dynamic pressure were chosen as $p_{m} / \rho a_{1}^{2}=-1.936$ $\times 10^{-3},-3.872 \times 10^{-3}$ and $\tau_{m}=5.093$.

In the process of computing, when the stress state at each mesh point enters the region $M^{*} \leqq \sqrt{2 J_{2}} / \sigma_{m}^{\prime}$, $\sqrt{2 J_{2}}=M^{*} \sigma_{m}^{\prime}$ was used assuming that the stress path moves along the critical state line under shear stress. When $\sigma_{k k} / 3>0, \sigma_{k k} / 3=-98.07 \mathrm{kPa}$ was assumed, whose value corresponds to the atomospheric pressure and it was used for convenience's sake to avoid $\sigma_{m}^{\prime}=$ 0 (See Eqs.(1), (2) and (17)).

\subsection{Numerical examples}

Figure 3 shows the dynamic pressure applied to the boundary of the cavity $\left(\alpha=\alpha_{1}\right)$. The ordinate shows the physical component of the stress tensor $g_{11} \mathrm{P}$. Figure 4 shows an example of numerical results at a point $(X=4.581$ and $Y=-4.185)$, which were

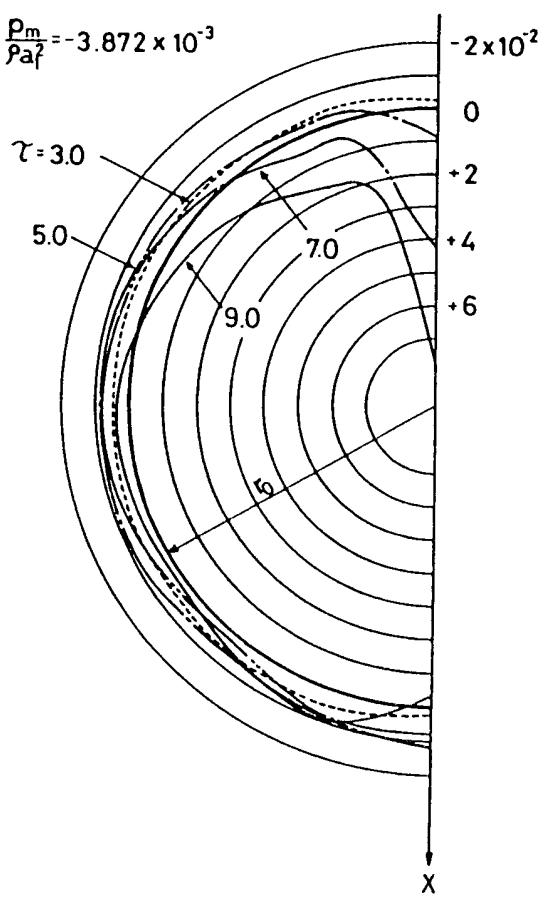

Fig. 5 Distributions of the physical component of the displacement $\sqrt{g_{11}} \int_{0}^{\tau} U d \tau$ along the bounday of the spherical cavity 


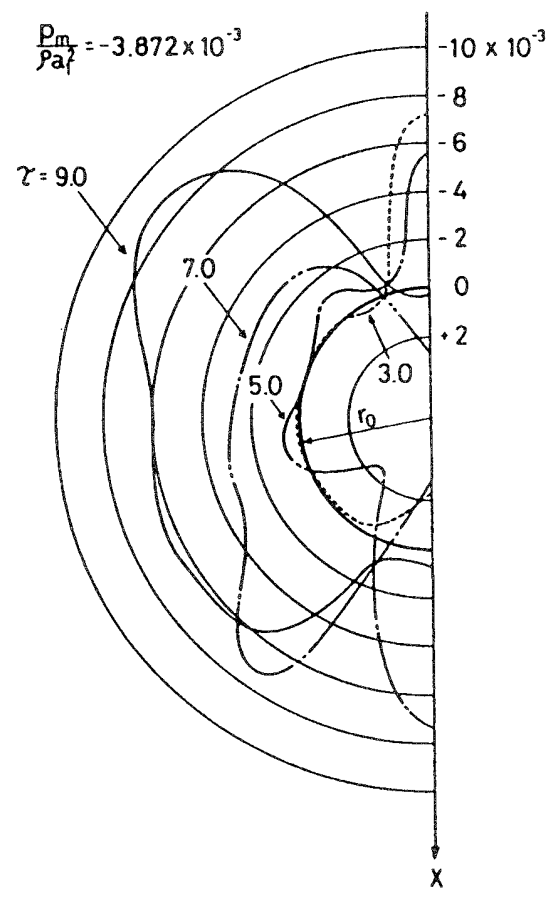

Fig. 6 Distributions of the physical component of the stress tensor $g_{11} \mathrm{Q}$ along the boundary of the spherical cavity

computed by changing the value of $\Delta \tau$, when $\Delta \alpha=\alpha_{1} /$ 30 and $\Delta \beta=\pi / 30$. We can observe in Fig. 4 that the solutions converge when $\Delta \tau=0.09 \sim 0.1$. The distributions of the physical component of the normal displacement on the boundary of the cavity are shown in Fig. 5, where the displacements are magnified, and therefore each curve does not show a real contour of the cavity at each time. The distributions of $g_{11} \mathrm{Q}$, the physical component of the stress tensor along the boundary of the cavity, are shown in Fig. 6. The bulging of the ground surface above the submerged cavity with time is shown in Fig. 7, by representing the distribution of the physical component of the displacement, $\sqrt{g_{11}} \int_{0}^{\tau} U d \tau$, along the surface.

\section{Concluding Remarks}

The governing equations of dynamic problems of the ground containing spherical cavities were for-

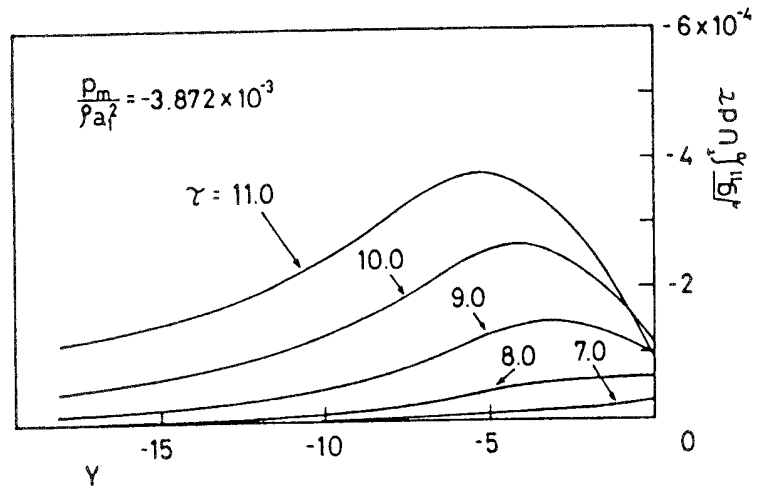

Fig. 7 Distributions of the physical component of the displacement along the ground surface

mulated in the system of bipolar coordinates, by modeling the soft ground of normally consolidated clays as an elastic-viscoplastic medium.

As numerical examples, the stress wave propagation was analyzed by the method of direct integration of the basic equations of the problem when axisymmetric dynamic loads were applied to the inside of the cavity. The soft ground is of fully saturated, normally consolidated clays which consist of solid and liquid phases. The changes in the ground pressure, the pore water pressure and the plastic volumetric strain with depth from the ground surface, in the initial state, were also taken into account. By carrying out the numerical computation, it was illustrated that the distributions of the stress and displacement on the boundary of the cavity and on the ground surface vary complicatedly with time $\tau$. The governing equations derived in this paper can be applied to analyzing dynamic problems in the soft ground containing cavities.

\section{References}

(1) Tanimura, S., Trans. Japan Soc. Mech. Eng. (in Japanese), Vol. 48, No. 434, A (1982-10), p. 1281.

(2) Tanimura, S., Ingenieur-Archiv, Vol. 52 (1982), p. 323.

(3) Adachi, T. and Oka, F., Soils and Foundations (Japanese Soc. Soil Mech. and Found. Eng.), Vol. 22, No. 4 (1982), p. 57. 